\title{
Prismatic Sets Associated with Planar Rooted Trees
}

\author{
S. Kaan Gürbüzer $\mathbb{D}^{1}$ and Bedia Akyar ${ }^{2}$ \\ ${ }^{1}$ Dokuz Eylül University, Department of Mathematics, Tinaztepe, Izmir, Turkey \\ ${ }^{2}$ Aalborg University, Department of Mathematical Sciences, Skjernvej 4, 9220 Aalborg Ø, Denmark \\ Correspondence should be addressed to S. Kaan Gürbüzer; kaan.gurbuzer@deu.edu.tr
}

Received 11 April 2019; Accepted 10 June 2019; Published 1 July 2019

Academic Editor: Li Guo

Copyright ( 2019 S. Kaan Gürbüzer and Bedia Akyar. This is an open access article distributed under the Creative Commons Attribution License, which permits unrestricted use, distribution, and reproduction in any medium, provided the original work is properly cited.

We construct the almost strong prismatic structure on the set of planar rooted trees and the bicomplex of planar rooted trees. Furthermore, we study the prismatic properties of Loday's algebraic operations on the set of planar rooted trees.

\section{Introduction}

Simplicial sets play an important role in simplicial category generated by two particularly important families of morphisms, whose images under given simplicial set functors are called face maps and degeneracy maps of those simplicial sets. Later, the concept of simplicial set was generalized by Dupont and Ljungman [1] by the name of prismatic sets which was derived from a simplicial complex, in order to construct an explicit fibre integration map in smooth Deligne cohomology [2]. Also, Akyar and Dupont [3] worked on the role of prismatic set in Lattice Gauge Field theory.

In his paper [4] about arithmetree, Loday introduced planar binary trees in homology theory related to dialgebras and constructed addition and multiplication operations on the set of planar binary rooted trees giving rise to a new kind of arithmetic theory. This theory has also been extended to all planar rooted trees. On the other hand, Frabetti [5] worked on the simplicial properties of the set of planar binary rooted trees and constructed a decomposition of the towers on the bicomplex related to planar binary rooted trees.

In this paper, we generalize the simplicial structure defined by Frabetti [5] of planar binary trees to the planar rooted trees. This structure is not simplicial but an almost simplicial structure called after Frabetti [5]. We construct an almost prismatic structure on the set of planar rooted trees and define a bicomplex on it. Furthermore, we investigate the combination of prismatic face and degeneracy maps with algebraic operations on trees defined by Loday [4].

\section{Preliminaries}

Definition 1. A simplicial set $S=\left\{S_{n}\right\}$ is a collection of sets $S_{n}$ with the face maps $d_{i}: S_{n} \longrightarrow S_{n-1}, 0 \leq i \leq n$ and degeneracy maps $s_{i}: S_{n} \longrightarrow S_{n+1}, 0 \leq i \leq n$ (with $n>0$ in the case of $d_{i}$ ) satisfying the simplicial identities
(d) $d_{i} d_{j}= \begin{cases}d_{j-1} d_{i}, & i<j ; \\ d_{j} d_{i+1}, & i \geq j\end{cases}$
(s) $s_{i} s_{j}= \begin{cases}s_{j+1} s_{i}, & i \leq j ; \\ s_{j} s_{i-1}, & i>j\end{cases}$
(ds) $d_{i} s_{j}= \begin{cases}s_{j-1} d_{i}, & i<j ; \\ i d, & i=j, j+1 ; \\ s_{j} d_{i-1}, & i>j+1 .\end{cases}$

Remark 2. In order to define the unique face map $d_{0}$ on degree zero, one can also append the empty set $\emptyset$ as $S_{-1}$ to a simplicial set. Instead of this, in this paper, we consider the face map $d_{0}$ on degree zero as the identity map on $S_{0}$.

One can weaken the conditions of simplicial sets. A collection of sets with only face maps satisfying the relation (d) is called a pre-simplicial set. A pseudo-simplicial set is a collection of sets with face and degeneracies satisfying the relations (d) and (ds). A subcollection of a pseudo-simplicial 

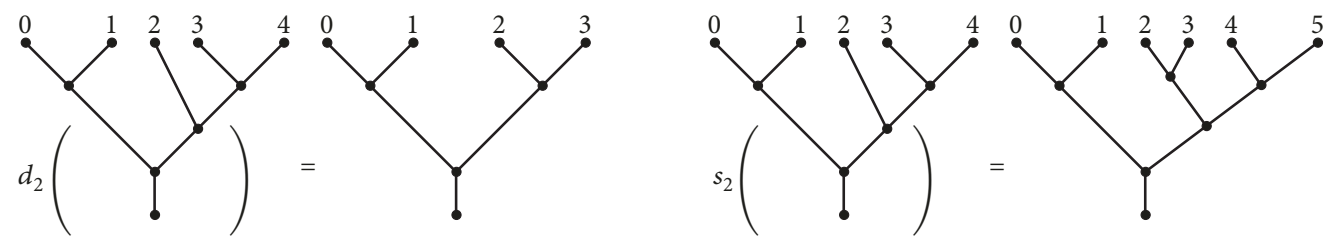

FIgURE 1: Examples of face and degeneracy maps $d_{2}, s_{2}$.

set whose degeneracies satisfy relation (s) except for $i=j$, that is, $s_{i} s_{i} \neq s_{i+1} s_{i}$, is called an almost simplicial set.

$$
\begin{aligned}
\{\text { simplicial sets }\} & \subset\{\text { almost simplicial sets }\} \\
& \subset\{\text { pseudo-simplicial sets }\} \\
& \subset\{\text { pre-simplicial sets }\}
\end{aligned}
$$

As given in Akyar [3], a strong prismatic set is a sequence $P:=\left\{P_{p}\right\}=\left\{S_{p, n_{0} n_{1} \ldots n_{p}}\right\}$ of $(p+1)$-multisimplicial sets together with face $d^{i}: S_{p, n_{0} \ldots n_{p}} \longrightarrow S_{p-1, n_{0} \ldots \widehat{n}_{i} \ldots n_{p}}$ and degeneracy $s^{i}:$ $S_{p, n_{0} \ldots n_{p}} \longrightarrow S_{p+1, n_{0} \ldots n_{i} n_{i} \ldots n_{p}}$ operators (with $p>0$ in the case of $d^{i}$ ) satisfying the following conditions:

$$
\begin{aligned}
& d^{i} d_{j}^{k}= \begin{cases}d_{j}^{k} d^{i}, & k<i \\
d^{i}, & k=i \\
d_{j}^{k-1} d^{i}, & k>i .\end{cases} \\
& d^{i} s_{j}^{k}= \begin{cases}s_{j}^{k} d^{i}, & k<i \\
d^{i}, & k=i \\
s_{j}^{k-1} d^{i}, & k>i .\end{cases} \\
& s^{i} d_{j}^{k}= \begin{cases}d_{j}^{k} s^{i}, & k<i \\
d_{j}^{k+1} d_{j}^{k} s^{i}, & k=i \\
d_{j}^{k+1} s^{i}, & k>i .\end{cases} \\
& s^{i} s_{j}^{k}= \begin{cases}s_{j}^{k} s^{i}, & k<i \\
s_{j}^{k+1} s_{j}^{k} s^{i}, & k=i \\
s_{j}^{k+1} s^{i}, & k>i,\end{cases}
\end{aligned}
$$

where the maps $d_{j}^{k}$ and $s_{j}^{k}$ are internal $j$-th face and degeneracy maps of the simplicial sets for each index $k$.

We recall that a graph $G$ is an ordered pairing $G=$ $(V(G), E(G))$ comprising the set $V(G)$ of vertices or nodes together with the set $E(G)$ of edges and a tree is a nonempty connected finite simple graph. Two vertices in a graph are called adjacent if and only if there exists an edge connecting them. If a vertex is adjacent to only one vertex in a graph then it is also called an external vertex. The nonexternal vertices are called internal vertices. Specially, a tree with a distinguished external vertex (root vertex) is called a rooted tree. For a rooted tree, the leaves are the external vertices different from the root. The degree or valency of an internal vertex is the number of edges incident to it. In this paper, we work on the planar rooted trees having at least one vertex and whose internal vertices have degree at least 3 . For any natural number $n$, we denote the set of all planar rooted trees with $n+1$ leaves by $T_{n}$. In particular, it can be set that $T_{-1}$ contains the unique planar rooted tree having only the root vertex. We define the degree of a planar rooted tree in $T_{n}$ by the number $n$. For a generic planar rooted tree in $T_{n}$, we assume that the leaves are labelled with the numbers, in the set $\{0, \ldots, n\}$ ordered from left to right and we denote it by $t_{n}$ to specify the degree of it.

In order to study on the simplicial properties of the collection of planar rooted trees, we consider the sequence $\mathscr{T}=\left\{T_{n}\right\}_{n \geq 0}$ of the sets of planar rooted trees with the face operators $d_{i}: T_{n} \longrightarrow T_{n-1}$ deleting the $i$-th leaf for $n>0$ and the degeneracy operators $s_{i}: T_{n} \longrightarrow T_{n+1}$ making a bifurcation on the $i$-th leaf for all $i \in\{0, \ldots n\}$, where $n \geq 0$. We give an example in Figure 1 of second face and degeneracy maps on $T_{4}$. The face and degeneracy maps satisfy the simplicial identities (d) and (s) except for the case $i=j$, that is, $s_{j} s_{j} \neq s_{j+1} s_{j}$. Let $t_{n} \in T_{n}$ and $i<j$, the $i$-th leaf of $t$ is again the $i$-th leaf of the tree $s_{j}\left(t_{n}\right)$, and if we apply first the face map $d_{i}$, the $j$-th leaf of $t_{n}$ becomes the $j-1$-st leaf of the tree $d_{i}\left(t_{n}\right)$, which means that the equality $d_{i} s_{j}=s_{j-1} d_{i}$ holds. When $i=j, j+1$, the bifurcation and deletion operations behave like inverses of each other. For the last case, if $i>j+1$, the face map $d_{i}$ deletes the $i$-th leaf of $s_{j}\left(t_{n}\right)$ which is the $i-1$ st leaf of $t_{n}$. So the equality $d_{i} s_{j}=s_{j} d_{i-1}$ also holds and hence the sequence $\mathscr{T}=\left\{T_{n}\right\}_{n \geq 0}$ is an almost simplicial set.

Definition 3. Combining the roots of two planar rooted trees on a vertex and creating a new root is called grafting operation and denoted by " $V$ ".

The grafting operation is not associative, that is, for different trees $t_{n_{1}}, t_{n_{2}}$, and $t_{n_{3}}$, the relation $\left(t_{n_{1}} \vee t_{n_{2}}\right) \vee t_{n_{3}}=$ $t_{n_{1}} \vee\left(t_{n_{2}} \vee t_{n_{3}}\right)$ is not satisfied and it is not commutative either. Without any confusion, we denote the grafting of more than two planar rooted trees by $t_{q_{0}} \vee \cdots \vee t_{q_{p}}$, where $t_{q_{i}} \in T_{q_{i}}$, and it is the planar rooted tree obtained by joining the roots of them to a new vertex and creating a new root; to imagine, see Figure 2. Note that the degree of the planar rooted tree $t_{q_{0}} \vee \cdots \vee t_{q_{p}}$ is $\sum_{i=0}^{p}\left(q_{i}+1\right)-1$. In this perspective, for any tree $t_{n} \in T_{n}$ with positive degree, there is a unique representation such that $t_{n}=t_{q_{0}} \vee \cdots \vee t_{q_{p}}$ where $t_{q_{i}} \in T_{q_{i}}$. For $n>0$, this allows us to decompose each set $T_{n}$ into the form $\bigcup_{q+p=n} T_{p, q_{0} \ldots q_{p}}$, where $1 \leq p \leq n, q_{i} \geq 0, \forall i, q=q_{0}+\cdots+q_{p}$ and $T_{p, q_{0} \ldots q_{p}}$ is the set of all possible planar rooted trees in the 


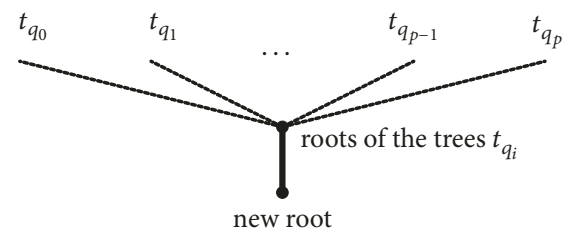

FIGURE 2: A general planar rooted tree of the form $t_{q_{0}} \vee \cdots \vee t_{q_{p}}$.

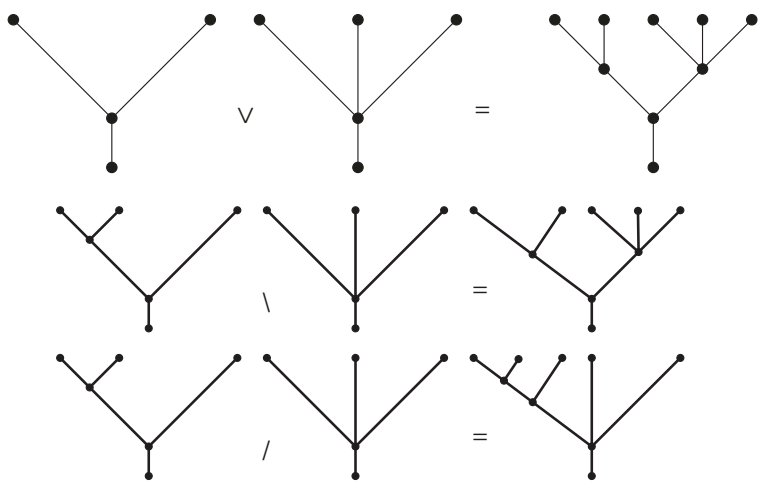

FIGURE 3: Example of grafting, over and under operations.

form $t_{q_{0}} \vee \cdots \vee t_{q_{p}}$ with $\sum_{i=0}^{p}\left(q_{i}+1\right)$ leaves. For $n=0$, we take attention that $p$ must be equal to 0 and hence $T_{0,0}=T_{0}$.

Let us recall the operations called over and under similar to the grafting from Loday [4]. The operation over is defined by grafting a tree to the most left leaf of another tree and similarly the operation under is defined by grafting a tree to the most right leaf of an another tree. For $t_{n_{1}} \in T_{n_{1}}$ and $t_{n_{2}} \in T_{n_{2}}, t_{n_{1}}$ over $t_{n_{2}}$ and $t_{n_{1}}$ under $t_{n_{2}}$ are denoted by $t_{n_{1}} / t_{n_{2}}$ and $t_{n_{1}} \backslash t_{n_{2}}$, respectively. Figure 3 helps us to imagine these operations.

In [4], Loday constructed the dendriform trialgebra of planar rooted trees with some operations such as the sum of two planar rooted trees recursively. Note that Loday defined the sum of two planar rooted trees as not a planar rooted tree but a grove which is a disjoint union of planar rooted trees. By definition, the unique planar rooted tree in $T_{0}$, denoted by 0 , can be considered as a unit element of the sum and for $k, l \geq 1$, the sum of two planar rooted trees $t_{n_{1}} \in T_{k, q_{0} \ldots q_{k}}$ and $t_{n_{2}} \in T_{l, r_{0} \ldots r_{l}}$ is explicitly given by a recursive formula,

$$
\begin{aligned}
t_{n_{1}}+t_{n_{2}}:= & t_{q_{0}} \vee \cdots \vee\left(t_{q_{k}}+t_{n_{2}}\right) \cup t_{q_{0}} \vee \cdots \\
& \vee\left(t_{q_{k}}+t_{r_{0}}\right) \vee \cdots \vee t_{r_{l}} \cup\left(t_{n_{1}}+t_{r_{0}}\right) \vee \cdots \\
& \vee t_{r_{l}},
\end{aligned}
$$

with the initial steps $0+0=0$ and

$$
\begin{aligned}
t_{1}+t_{1} & =0 \vee\left(0+t_{1}\right) \cup 0 \vee(0+0) \vee 0 \cup\left(t_{1}+0\right) \vee 0 \\
& =0 \vee(0 \vee 0) \cup 0 \vee 0 \vee 0 \cup(0 \vee 0) \vee 0,
\end{aligned}
$$

where $t_{1}=0 \vee 0$ is the unique planar rooted tree with two leaves in $T_{1}$.
In this formula, the disjoint union operation is used to obtain the grove and the grafting operation is considered as distribution on the disjoint union. Loday separated this sum into three parts and considered each part as a result of another sum. These three sums are called left sum $\dashv$, middle sum $\perp$ and right sum $\vdash$ and defined by

$$
\begin{aligned}
\dashv: & T_{k, q_{0} \ldots q_{k}} \times T_{l, r_{0} \ldots r_{l}} \\
& \longrightarrow \mathbb{T}_{k, q_{0} \ldots q_{k-1}}\left(q_{k}+r_{0}+\ldots r_{l}+l\right), \\
t_{n_{1}} & \dashv t_{n_{2}}=t_{q_{0}} \vee \cdots \vee\left(t_{q_{k}}+t_{2}\right), \\
\perp: T_{k, q_{0} \ldots q_{k}} \times T_{l, r_{0} \ldots r_{l}} & \longrightarrow \mathbb{T}_{k+l, q_{0} \ldots q_{k-1}\left(q_{k}+r_{0}\right) r_{1} \ldots r_{l}}, \\
t_{n_{1}} \perp t_{n_{2}} & =t_{q_{0}} \vee \cdots \vee\left(t_{q_{k}}+t_{r_{0}}\right) \vee \cdots \vee t_{r_{l}}, \\
& \vdash: T_{k, q_{0} \ldots q_{k}} \times T_{l, r_{0} \ldots r_{l}} \\
& \longrightarrow \mathbb{T}_{l,\left(q_{0}+\cdots+q_{k}+k+r_{0}\right) r_{1} \ldots r_{l}}, \\
t_{n_{1}} & \vdash t_{n_{2}}=\left(t_{1}+t_{r_{0}}\right) \vee \cdots \vee t_{r_{l}},
\end{aligned}
$$

respectively, where $t_{n_{1}} \neq 0$ and $t_{n_{2}} \neq 0$ and $\mathbb{T}_{p, q_{0} \ldots q_{p}}$ denotes the set of groves, that is, the set of all disjoint union of the planar rooted trees of the form $t_{q_{0}} \vee \cdots \vee t_{q_{p}}$ with degree $\left(\sum_{i=0}^{p} q_{p}\right)+p$. The extension of these sums for 0 is given by $t_{n_{1}} \dashv 0=t_{n_{1}}, 0 \dashv t_{n_{2}}=0, t_{n_{1}} \vdash 0=0,0 \vdash t_{n_{2}}=t_{n_{2}}$, and $t_{n_{1}} \perp 0=0,0 \perp t_{n_{2}}=0$.

\section{Main Results}

Theorem 4. For any field $F$, let $F\left[T_{n}\right]$ be the vector space over $F$ with basis $T_{n}$, the sequence $0 \longleftarrow F\left[T_{0}\right] \longleftarrow F\left[T_{1}\right] \longleftarrow \cdots \longleftarrow$ $F\left[T_{n}\right] \longleftarrow \cdots$ is a chain complex $F\left[T_{*}\right]$ with the boundary operator $\partial=\sum_{i=0}^{n}(-1)^{i} d_{i}$.

Theorem 5. For any field $F$, the chain complex $F\left[T_{*}\right]$ is acyclic, that is, $H_{n}\left(F\left[T_{*}\right], \partial\right)=\{F, n=0 ; 0, n \neq 0\}$.

In order to construct a bicomplex on the set of all planar rooted trees, we observe an almost strong prismatic structure of the collection $\mathscr{T}$. The decomposition of $T_{n}$ given in previous section gives us the following theorem.

Theorem 6. The collection of all planar rooted trees becomes an almost strong prismatic set with the decomposition

$$
\mathscr{T}=\bigcup_{n} \bigcup_{q+p=n} T_{p, q_{0} \ldots q_{p}},
$$

where the face maps $d^{k}: T_{p, q_{0} \ldots q_{p}} \longrightarrow T_{p-1, q_{0} \ldots \widehat{q_{k}} \ldots q_{p}}$ deleting the $k$-th tree for $p>0$ and the degeneracies $s^{k}: T_{p, q_{0} \ldots q_{p}} \longrightarrow$ $T_{p+1, q_{0} \ldots q_{k} q_{k} \ldots q_{p}}$ repeating the $k$-th tree; that is,

$$
\begin{gathered}
d^{k}\left(t_{q_{0}} \vee \cdots \vee t_{q_{p}}\right)= \\
s^{k}\left(t_{q_{0}} \vee \cdots \vee t_{q_{p}}\right)= \\
t_{q_{0}} \vee \cdots \vee t_{q_{k-1}} \vee t_{q_{k+1}} \vee \cdots \vee t_{q_{k-1}} \vee t_{q_{k}} \vee t_{q_{k}} \vee t_{q_{k+1}} \\
\vee \cdots \vee t_{q_{p}} .
\end{gathered}
$$


The internal face and degeneracy maps are the face and degeneracy maps given in the almost simplicial structure.

Theorem 7. For $t_{n_{1}} \in T_{k, q_{0} \ldots q_{k}}$ and $t_{n_{2}} \in T_{l, r_{0} \ldots r_{l}}$, prismatic face and degeneracy maps have effect to over and under operations as follows:

$$
\begin{aligned}
& d^{i}\left(t_{n_{1}} / t_{n_{2}}\right)= \begin{cases}d^{i} t_{n_{2}}, & i=0 ; \\
t_{n_{1}} / d^{i} t_{n_{2}}, & i \neq 0 .\end{cases} \\
& d^{i}\left(t_{n_{1}} \backslash t_{n_{2}}\right)= \begin{cases}d^{i} t_{n_{1}}, & i=k ; \\
d^{i} t_{n_{1}} \backslash t_{n_{2}}, & i \neq k .\end{cases} \\
& s^{i}\left(t_{n_{1}} / t_{n_{2}}\right)
\end{aligned}
$$

$$
\begin{aligned}
& = \begin{cases}\left(t_{n_{1}} / t_{r_{0}}\right) \vee\left(t_{n_{1}} / t_{r_{0}}\right) \vee t_{r_{1}} \vee \cdots \vee t_{r_{l}}, & i=0 ; \\
t_{n_{1}} / s^{i} t_{n_{2}}, & i \neq 0 .\end{cases} \\
& s^{i}\left(t_{n_{1}} \backslash t_{n_{2}}\right) \\
& = \begin{cases}t_{q_{0}} \vee \cdots \vee\left(t_{q_{k}} \backslash t_{n_{2}}\right) \vee\left(t_{q_{k}} \backslash t_{n_{2}}\right), & i=k ; \\
s^{i} t_{n_{1}} \backslash t_{n_{2}}, & i \neq k .\end{cases}
\end{aligned}
$$

Theorem 8. For $t_{n_{1}} \in T_{k, q_{0} \ldots q_{k}}$ and $t_{n_{2}} \in T_{l, r_{0} \ldots r_{l}}$ different from 0 , the effects of prismatic external face and degeneracy maps on these tree sum operations are given as follows:

$$
\begin{aligned}
& d^{i}\left(t_{n_{1}} \dashv t_{n_{2}}\right)= \begin{cases}d^{i} t_{n_{1}} \dashv t_{n_{2}}, & i \neq k \\
d^{k} t_{n_{1}}, & i=k\end{cases} \\
& d^{i}\left(t_{n_{1}} \perp t_{n_{2}}\right)= \begin{cases}d^{i} t_{n_{1}} \perp t_{n_{2}}, & i<k \\
t_{q_{0}} \vee \cdots \vee t_{q_{k-1}} \vee t_{r_{1}} \vee \cdots \vee t_{r_{l}}, & i=k \\
t_{n_{1}} \perp d^{i-k} t_{n_{2}}, & i>k .\end{cases} \\
& d^{i}\left(t_{n_{1}} \vdash t_{n_{2}}\right)= \begin{cases}d^{0} t_{n_{2}}, & i=0 \\
t_{n_{1}} \vdash d^{i} t_{n_{2}}, & i \neq 0 .\end{cases} \\
& s^{i}\left(t_{n_{1}} \dashv t_{n_{2}}\right)= \begin{cases}s^{i} t_{n_{1}} \dashv t_{n_{2}}, & i \neq k \\
t_{q_{0}} \vee \cdots \vee t_{q_{k-1}} \vee\left(t_{q_{k}}+t_{n_{2}}\right) \vee\left(t_{q_{k}}+t_{n_{2}}\right), & i=k .\end{cases} \\
& s^{i}\left(t_{n_{1}} \perp t_{n_{2}}\right)= \begin{cases}s^{i} t_{n_{1}} \perp t_{n_{2}}, & i<k \\
t_{q_{0}} \vee \cdots \vee t_{q_{k-1}} \vee\left(t_{q_{k}}+t_{r_{0}}\right) \vee\left(t_{q_{k}}+t_{r_{0}}\right) \vee t_{r_{1}} \vee \cdots \vee t_{r_{1}}, & i=k \\
t_{n_{1}} \perp s^{i-k} t_{n_{2}}, & i>k .\end{cases} \\
& s^{i}\left(t_{n_{1}} \vdash t_{n_{2}}\right)= \begin{cases}t_{n_{1}} \vdash s^{i} t_{n_{2}}, & i \neq 0 ; \\
\left(t_{n_{1}}+t_{r_{0}}\right) \vee\left(t_{n_{1}}+t_{r_{0}}\right) \vee t_{r_{1}} \vee \cdots \vee t_{r_{1}}, & i=0 .\end{cases}
\end{aligned}
$$

Taking a symmetry on the axes passing through the root edge gives an involution on the set of trees. We denote this involution by $\sigma$ and it is clear that there is a distribution property of the involution on grafting and hence the sums of trees. For $t_{n} \in T_{k, q_{0} \ldots q_{k}}$, it can be checked that the relations $d^{i} \sigma\left(t_{n}\right)=\sigma\left(d^{k-i} t_{n}\right)$ and $s^{i} \sigma\left(t_{n}\right)=\sigma\left(s^{k-i} t_{n}\right)$ hold.

The prismatic structure of the collection $\mathscr{T}$ allows us to divide the chain complex $F\left[T_{*}\right]$, given in Theorem 4, into two parts called horizontal and vertical.

The boundary operators $\partial_{v}^{k}: F\left[T_{p, q_{0} \ldots q_{k} \ldots q_{p}}\right] \longrightarrow$ $F\left[T_{p, q_{0} \ldots q_{k}-1 \ldots q_{p}}\right]$ of the vertical complex are defined by $\partial_{v}^{k}=0$ whenever $q_{k}=0$; otherwise $\partial_{v}^{k}=\sum_{j=0}^{q_{k}}(-1)^{j} d_{j}^{k}$. The total boundary operator of the vertical complexes is given by

$$
\partial_{v}:=\partial_{v}^{0}+(-1)^{q_{0}+1} \partial_{v}^{1}+\cdots+(-1)^{q_{0}+\cdots+q_{p-1}+p} \partial_{v}^{p} .
$$

For each horizontal complex, the boundary operator $\partial_{h}$ : $F\left[T_{p, q_{0} \ldots q_{p}}\right] \longrightarrow F\left[T_{p-1, q_{0}^{\prime} \ldots q_{p-1}^{\prime}}\right]$ is defined by

$$
\partial_{h}=\partial_{0}+(-1)^{q_{0}+1} \partial_{1}+\cdots+(-1)^{q_{0}+\cdots+q_{p-1}+p} \partial_{p}
$$

where $\partial_{i}=\left\{0\right.$, if $q_{i}>0 ; d^{i}$, if $\left.q_{i}=0\right\}$.

Theorem 9. The vertical and horizontal boundary operators satisfy the identities $\partial_{v} \partial_{v}=0$ and $\partial_{h} \partial_{h}=0$. For $p>0$ and $q=$ $q_{0}+q_{1}+\cdots+q_{p}$, the complexes $\left(F\left[T_{*, q}\right], \partial_{h}\right)$ and $\left(F\left[T_{p, *}\right], \partial_{v}\right)$ are chain complexes. In addition, the triple $\left(F\left[T_{*, *}\right], \partial_{v}, \partial_{h}\right)$ forms a chain bicomplex as shown in Figure 4 and the total complex $F\left[T_{*}\right]$ is given by the boundary operator $\widetilde{\partial}=\partial_{v}+\partial_{h}$. 


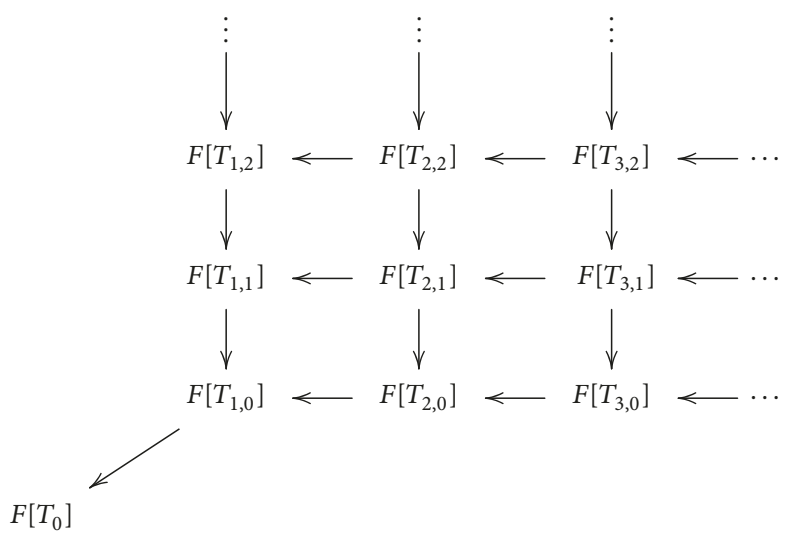

FIgURE 4: The bicomplex of planar rooted trees.

Corollary 10. For any $t_{n_{1}} \in T_{n_{1}}$ and $t_{n_{2}} \in T_{n_{2}}$, the vertical and horizontal boundary operators satisfy the followings

$$
\begin{aligned}
& \partial_{v}\left(t_{n_{1}} \vee t_{n_{2}}\right)=\partial t_{n_{1}} \vee t_{n_{2}}+(-1)^{n_{1}+1} t_{n_{1}} \vee \partial t_{n_{2}}, \\
& \partial_{h}\left(t_{n_{1}} \vee t_{n_{2}}\right)= \begin{cases}0, & n_{1}=n_{2}=0 \\
t_{n_{2}}, & n_{1}=0 \text { and } n_{2} \neq 0 \\
(-1)^{n_{1}+1} t_{n_{1}}, & n_{1} \neq 0 \text { and } n_{2}=0 \\
0, & n_{1} \neq 0 \text { and } n_{2} \neq 0\end{cases}
\end{aligned}
$$

Furthermore, the relation $\widetilde{\partial}\left(t_{n_{1}} \vee t_{n_{2}}\right)=\partial t_{n_{1}} \vee t_{n_{2}}+(-1)^{n_{1}+1} t_{n_{1}} \vee$ $\partial t_{n_{2}}$ holds.

\subsection{Proofs}

Proof of Theorem 4. Since the effects of the maps $d_{i} d_{j}$ and $d_{j-1} d_{i}$ are the same for $i<j$, the boundary operator satisfies the condition $\partial^{2}=0$ and hence the sequence becomes a chain complex.

Proof of Theorem 5. Let us define a map $h: T_{n} \longrightarrow T_{n+1}$ which grafts a leaf to the root edge; that is, for $t_{n} \in T_{n}, h\left(t_{n}\right)=0 \vee t_{n}$. For any $j>0$, it can be seen that the relation $d_{j} h=h d_{j-1}$ is satisfied and the composition $d_{0} h$ becomes identity. Then, we get

$$
\begin{aligned}
\partial h & =\sum_{i=0}^{n+1}(-1)^{i} d_{i} \circ h=d_{0} h+\sum_{i=1}^{n+1}(-1)^{i} d_{i} h \\
& =d_{0} h+\sum_{i=0}^{n}(-1)^{i+1} d_{i+1} h=d_{0} h-\sum_{i=0}^{n}(-1)^{i} d_{i+1} h \\
& =d_{0} h-\sum_{i=0}^{n}(-1)^{i} h d_{i}=d_{0} h-h \partial .
\end{aligned}
$$

So, $\partial h+h \partial=$ identity and the induced map $h: F\left[T_{n}\right] \longrightarrow$ $F\left[T_{n+1}\right]$ becomes a homotopy between identity and zero map.
Proof of Theorem 6. First we check that the simplicial identities are satisfied for external face and degeneracy maps. For $t_{n}=t_{q_{0}} \vee \cdots \vee t_{q_{p}}$

$$
\begin{aligned}
d^{i} d^{j}\left(t_{n}\right)= & d^{i}\left(t_{q_{0}} \vee \cdots \vee t_{q_{j-1}} \vee t_{q_{j+1}} \vee \cdots \vee t_{q_{p}}\right) \\
= & t_{q_{0}} \vee \cdots \vee t_{q_{i-1}} \vee t_{q_{i+1}} \vee \cdots \vee t_{q_{j-1}} \vee t_{q_{j+1}} \\
& \vee \cdots \vee t_{q_{p}} \\
= & d^{j-1}\left(t_{q_{0}} \vee \cdots \vee t_{q_{i-1}} \vee t_{q_{i+1}} \vee \cdots \vee t_{q_{p}}\right) \\
= & d^{j-1} d^{i}\left(t_{n}\right), \quad \text { for } i<j ;
\end{aligned}
$$

$$
\begin{aligned}
& d^{i} d^{j}\left(t_{n}\right)=d^{i}\left(t_{q_{0}} \vee \cdots \vee t_{q_{j-1}} \vee t_{q_{j+1}} \vee \cdots \vee t_{q_{p}}\right) \\
& =t_{q_{0}} \vee \cdots \vee t_{q_{j-1}} \vee t_{q_{j+1}} \vee \cdots \vee t_{q_{i}} \vee t_{q_{i+2}} \\
& \vee \cdots \vee t_{q_{p}} \\
& =d^{j}\left(t_{q_{0}} \vee \cdots \vee t_{q_{i}} \vee t_{q_{i+2}} \vee \cdots \vee t_{q_{p}}\right) \\
& =d^{j} d^{i+1}\left(t_{n}\right), \quad \text { for } i \geq j \text {; } \\
& s^{i} s^{j}\left(t_{n}\right)=s^{i}\left(t_{q_{0}} \vee \cdots \vee t_{q_{j}} \vee t_{q_{j}} \vee \cdots \vee t_{q_{p}}\right) \\
& =t_{q_{0}} \vee \cdots \vee t_{q_{i}} \vee t_{q_{i}} \vee \cdots \vee t_{q_{j}} \vee t_{q_{j}} \vee \cdots \\
& \vee t_{q_{p}} \\
& =s^{j+1}\left(t_{q_{0}} \vee \cdots \vee t_{q_{i}} \vee t_{q_{i}} \vee \cdots \vee t_{q_{p}}\right) \\
& =s^{j+1} s^{i}\left(t_{n}\right), \quad \text { for } i<j \text {; } \\
& s^{i} s^{j}\left(t_{n}\right)=s^{i}\left(t_{q_{0}} \vee \cdots \vee t_{q_{j}} \vee t_{q_{j}} \vee \cdots \vee t_{q_{p}}\right) \\
& =t_{q_{0}} \vee \cdots \vee t_{q_{j}} \vee t_{q_{j}} \vee \cdots \vee t_{q_{i-1}} \vee t_{q_{i-1}} \vee \cdots \\
& \vee t_{q_{p}} \\
& =s^{i-1}\left(t_{q_{0}} \vee \cdots \vee t_{q_{j}} \vee t_{q_{j}} \vee \cdots \vee t_{q_{p}}\right) \\
& =s^{j} s^{i-1}\left(t_{n}\right), \quad \text { for } i>j \text {; } \\
& s^{i} s^{i}\left(t_{n}\right)=s^{i}\left(t_{q_{0}} \vee \cdots \vee t_{q_{i}} \vee t_{q_{i}} \vee \cdots \vee t_{q_{p}}\right) \\
& =t_{q_{0}} \vee \cdots \vee t_{q_{i}} \vee t_{q_{i}} \vee t_{q_{i}} \vee \cdots \vee t_{q_{p}} \\
& =s^{i+1}\left(t_{q_{0}} \vee \cdots \vee t_{q_{i}} \vee t_{q_{i}} \vee \cdots \vee t_{q_{p}}\right) \\
& =s^{i+1} s^{i}\left(t_{n}\right) \text {. }
\end{aligned}
$$

Now, we check the identities (3) and (4).

$$
\begin{aligned}
d^{i} d_{j}^{k}\left(t_{n}\right) & =d^{i}\left(t_{q_{0}} \vee \cdots \vee d_{j}\left(t_{q_{k}}\right) \vee \cdots \vee t_{q_{p}}\right) \\
& =t_{q_{0}} \vee \cdots \vee d_{j}\left(t_{q_{k}}\right) \vee \cdots \vee t_{q_{i-1}} \vee t_{q_{i+1}} \vee \cdots
\end{aligned}
$$




$$
\begin{aligned}
& \vee t_{q_{p}}=d_{j}^{k} d^{i}\left(t_{n}\right) ; \text { for } k<i ; \\
& d^{i} d_{j}^{k}\left(t_{n}\right)= d^{i}\left(t_{q_{0}} \vee \cdots \vee d_{j}\left(t_{q_{k}}\right) \vee \cdots \vee t_{q_{p}}\right) \\
&= t_{q_{0}} \vee \cdots \vee t_{q_{i-1}} \vee t_{q_{i+1}} \vee \cdots \vee t_{q_{p}}=d^{i}\left(t_{n}\right) ; \\
& \text { for } k=i ; \\
& d^{i} d_{j}^{k}\left(t_{n}\right)= d^{i}\left(t_{q_{0}} \vee \cdots \vee d_{j}\left(t_{q_{k}}\right) \vee \cdots \vee t_{q_{p}}\right) \\
&= t_{q_{0}} \vee \cdots \vee t_{q_{i-1}} \vee t_{q_{i+1}} \vee \cdots \vee d_{j}\left(t_{q_{k}}\right) \vee \cdots \\
& \vee t_{q_{p}}=d_{j}^{k-1} d^{i}\left(t_{n}\right) ; \quad \text { for } k>i ; \\
& s^{i} d_{j}^{k}\left(t_{n}\right)= s^{i}\left(t_{q_{0}} \vee \cdots \vee d_{j}\left(t_{q_{k}}\right) \vee \cdots \vee t_{q_{p}}\right) \\
&= t_{q_{0}} \vee \cdots \vee d_{j}\left(t_{q_{k}}\right) \vee \cdots \vee t_{q_{i}} \vee t_{q_{i}} \vee \cdots \\
& \vee t_{q_{p}}=d_{j}^{k} s^{i}\left(t_{n}\right) ; \quad \text { for } k<i ; \\
& s^{i} d_{j}^{k}\left(t_{n}\right)= s^{i}\left(t_{q_{0}} \vee \cdots \vee d_{j}\left(t_{q_{k}}\right) \vee \cdots \vee t_{q_{p}}\right) \\
&= t_{q_{0}} \vee \cdots \vee d_{j}\left(t_{q_{k}}\right) \vee d_{j}\left(t_{q_{k}}\right) \vee \cdots \vee t_{q_{p}} \\
&= d_{j}^{k+1} d_{j}^{k} s^{i}\left(t_{n}\right) ; \quad \text { for } k=i ; \\
& s^{i} d_{j}^{k}\left(t_{n}\right)= s^{i}\left(t_{q_{0}} \vee \cdots \vee d_{j}\left(t_{q_{k}}\right) \vee \cdots \vee t_{q_{p}}\right) \\
&= t_{q_{0}} \vee \cdots \vee t_{q_{i}} \vee t_{q_{i}} \vee \cdots \vee d_{j}\left(t_{q_{k}}\right) \vee \cdots \\
& \vee t_{q_{p}}=d_{j}^{k+1} s^{i}\left(t_{n}\right) ; \quad \text { for } k>i .
\end{aligned}
$$

The prismatic identities between external maps and internal degeneracies can be verified in a similar way.

Proof of Theorem 9. To simplify the notation, let $Q_{k}$ denote the sum $q_{0}+\cdots+q_{k-1}+k$ for $k>0$ and $Q_{0}=0$. For $l<k$ and $q_{k} \neq 0 \neq q_{l}$, in the composition $\partial_{v} \partial_{v}$, we have the sum

$$
(-1)^{Q_{k}^{\prime}}(-1)^{Q_{l}} \partial_{v}^{k} \partial_{v}^{l}+(-1)^{Q_{l}^{\prime}}(-1)^{Q_{k}} \partial_{v}^{l} \partial_{v}^{k},
$$

which is equal to 0 , because after applying $\partial_{v}^{l}$, the index of the $l$-th part of the planar rooted tree becomes $q_{l}-1$ and so $Q_{k}^{\prime}=Q_{k}-1$ and $Q_{l}=Q_{l}^{\prime}$. For the case $l=k$, the proof induces the boundary operator $\partial$ given in Theorem 4 on the $k$-th part of the planar rooted tree and hence $\partial_{v} \partial_{v}=0$.

In order to prove that $\partial_{h} \partial_{h}=0$, we only need to consider the planar rooted trees which have 0 at least for two indices. Now, we assume that $l<k$ and $q_{l}=q_{k}=0$. In this case, for the composition $\partial_{h} \partial_{h}$ includes the sums of the form

$$
(-1)^{Q_{l}^{\prime}}(-1)^{Q_{k}} \partial_{l} \partial_{k}+(-1)^{Q_{k-1}^{\prime}}(-1)^{Q_{l}} \partial_{k-1} \partial_{l} .
$$

Since $q_{l}=0$, we obtain that $Q_{k-1}^{\prime}=Q_{k}-1$ and $Q_{l}^{\prime}=Q_{l}$. Hence this sum is equal to 0 and $\partial_{h} \partial_{h}=0$.
Last, similarly the composition $\partial_{h} \partial_{v}$ can be rewritten by shifting one the indices of the composition $\partial_{v} \partial_{h}$ and hence the equality $\widetilde{\partial} \widetilde{\partial}=0$ holds.

\section{Concluding Remarks}

Loday [6] and Frabetti [7] used the bicomplex on the collection of the sets of planar binary rooted trees to construct the homology and cohomology theory of dialgebras. In order to study on the homology or cohomology theory on dendriform trialgebras, the prismatic structure and the bicomplex given in this paper may be a useful tool.

\section{Data Availability}

No data were used to support this study.

\section{Conflicts of Interest}

The author declares that there are no conflicts of interest regarding the publication of this paper.

\section{References}

[1] J. L. Dupont and R. Ljungmann, "Integration of simplicial forms and Deligne cohomology," Mathematica Scandinavica, vol. 97, no. 1, pp. 11-39, 2005.

[2] J. L. Dupont and F. W. Kamber, "On a generalization of CHEeger-CHErn-Simons classes," Illinois Journal of Mathematics, vol. 34, no. 2, pp. 221-255, 1990.

[3] B. Akyar and J. L. Dupont, "Lattice gauge field theory and prismatic sets," Mathematica Scandinavica, vol. 108, no. 1, pp. 26-54, 2011.

[4] J.-L. Loday, "Arithmetree," Journal of Algebra, vol. 258, no. 1, pp. 275-309, 2002.

[5] A. Frabetti, "Simplicial properties of the set of planar binary trees," Journal of Algebraic Combinatorics. An International Journal, vol. 13, no. 1, pp. 41-65, 2001.

[6] J.-L. Loday, "Dialgebras," in Dialgebras and Related Operads, vol. 1763 of Lecture Notes in Mathematics, pp. 7-66, Springer, Berlin, Germany, 2001.

[7] A. Frabetti, "Dialgebra (co)homology with coefficients," in Dialgebras and related operads, vol. 1763 of Lecture Notes in Math., pp. 67-103, Springer, Heidelberg, Berlin, 2001. 


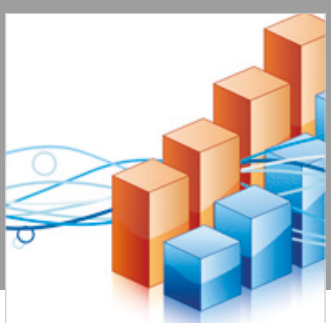

Advances in

Operations Research

\section{-n-m}
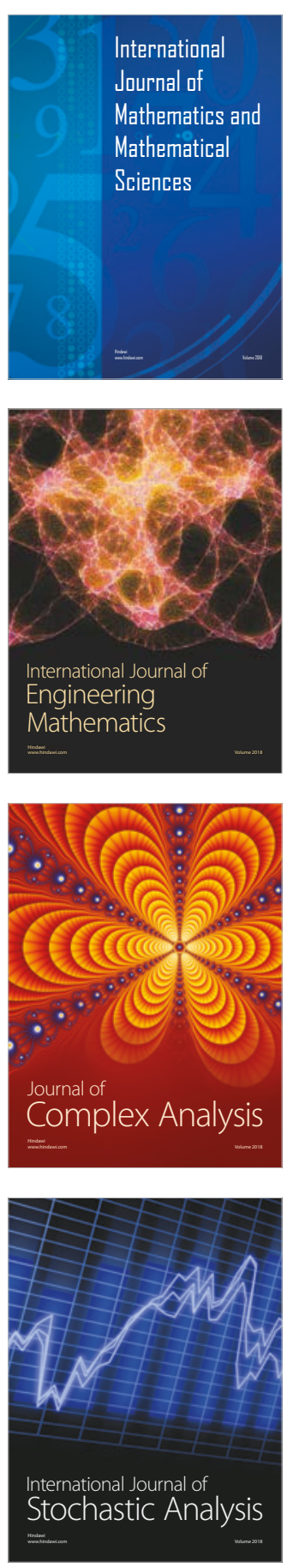
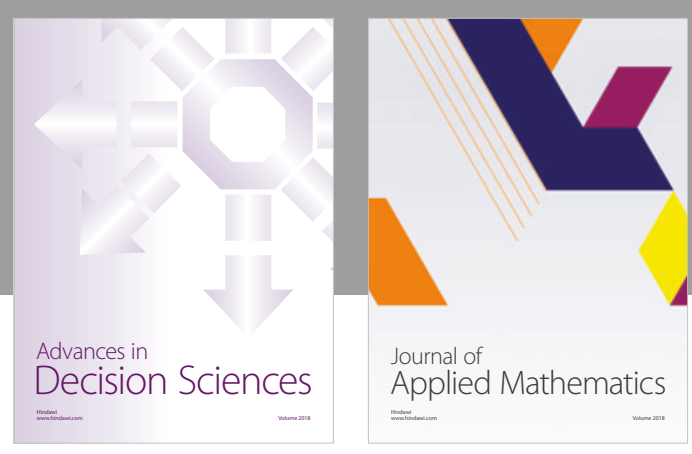

Journal of

Applied Mathematics
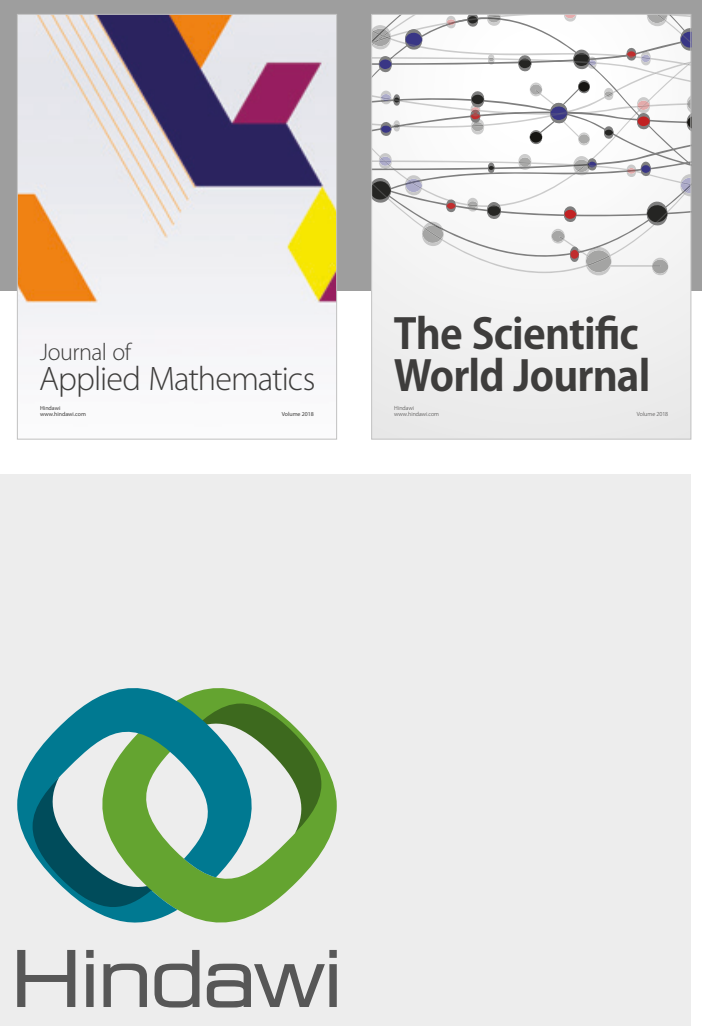

Submit your manuscripts at

www.hindawi.com

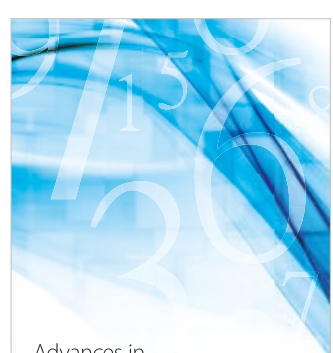

Advances in
Numerical Analysis
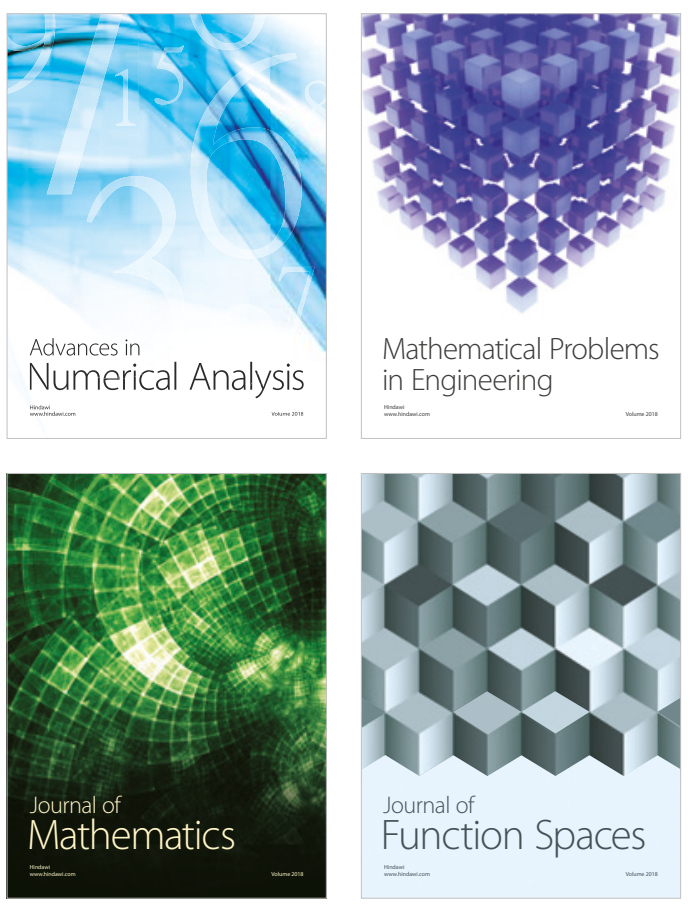

Mathematical Problems in Engineering

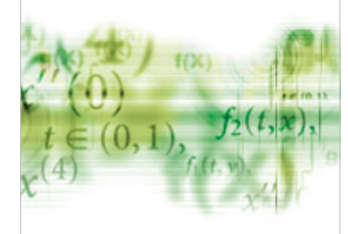

International Journal of

Differential Equations

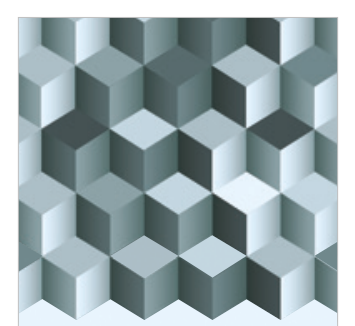

Journal of

Function Spaces
The Scientific

World Journal

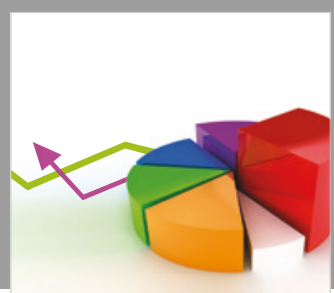

Journal of

Probability and Statistics
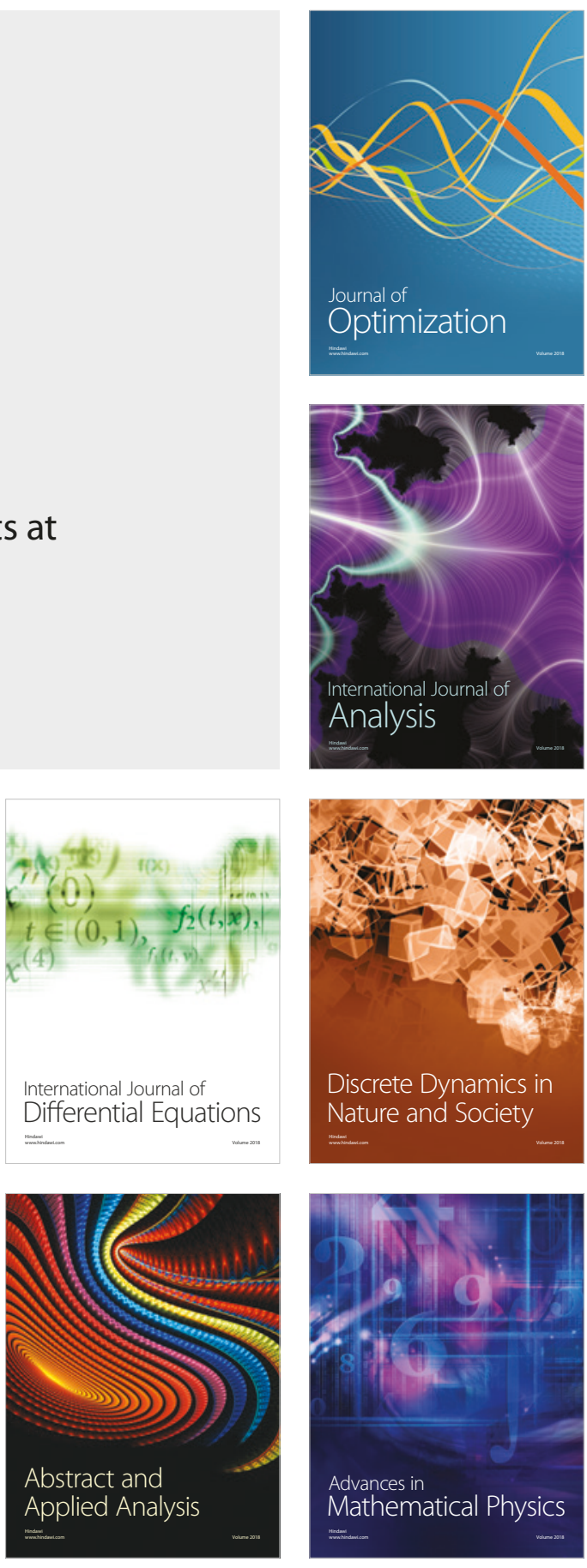